Supplement of Biogeosciences, 13, 3981-3989, 2016

http://www.biogeosciences.net/13/3981/2016/

doi:10.5194/bg-13-3981-2016-supplement

(C) Author(s) 2016. CC Attribution 3.0 License.

(c) (i)

Supplement of

\title{
Surfactant control of gas transfer velocity along an offshore coastal tran- sect: results from a laboratory gas exchange tank
}

\section{R. Pereira et al.}

Correspondence to: R. Pereira (r.pereira@hw.ac.uk)

The copyright of individual parts of the supplement might differ from the CC-BY 3.0 licence. 


\section{Supplement}

Table S1: Surfactant activity (normalized to Triton T-X-100), total CDOM absorbance (250-450 nm), $\mathrm{S}_{275-295}, \mathrm{~S}_{350-400}, S_{R}, E_{2}$ : $E_{3}$ ratio and SA enrichment factor (EF) of the sea surface microlayer (SML) from a North Sea transect during 2012-2013.

\begin{tabular}{|c|c|c|c|c|c|c|c|c|}
\hline Date & $\begin{array}{c}\text { Dove } \\
\text { Station }\end{array}$ & $\begin{array}{c}\text { SML SA } \\
\mathrm{mg} \mathrm{l}^{-1} \\
\mathrm{~T}-\mathrm{X}-100\end{array}$ & $\begin{array}{c}\text { SML } \\
\text { CDOM }_{250-} \\
450 \\
\text { Total } a\end{array}$ & $\begin{array}{c}\text { SML } \\
S_{275-295} \\
\mathrm{~nm}^{-1}\end{array}$ & $\begin{array}{c}\text { SML } \\
S_{350-400} \\
\mathrm{~nm}^{-1}\end{array}$ & SML $S_{R}$ & $\begin{array}{c}\text { SML } \\
E_{2}: E_{3}\end{array}$ & SA EF \\
\hline $04 / 10 / 12$ & 1 & $\begin{array}{c}0.27 \\
\pm 0.03\end{array}$ & $\begin{array}{l}874.8 \\
\pm 37.6\end{array}$ & $\begin{array}{c}0.013 \\
\pm 0.001\end{array}$ & $\begin{array}{c}0.010 \\
\pm 0.001\end{array}$ & $\begin{array}{c}1.32 \\
\pm 0.01\end{array}$ & $\begin{array}{c}3.580 \\
\pm 0.250\end{array}$ & $\begin{array}{c}1.44 \\
\pm 0.40\end{array}$ \\
\hline $04 / 10 / 12$ & 2 & $\begin{array}{c}0.22 \\
\pm 0.08\end{array}$ & $\begin{array}{c}429.0 \\
\pm 156.7\end{array}$ & $\begin{array}{c}0.016 \\
\pm 0.002\end{array}$ & $\begin{array}{c}0.008 \\
\pm 0.004\end{array}$ & $\begin{array}{c}1.99 \\
\pm 0.01\end{array}$ & $\begin{array}{c}4.291 \\
\pm 1.026\end{array}$ & $\begin{array}{c}1.90 \\
\pm 0.71\end{array}$ \\
\hline $04 / 10 / 12$ & 3 & $\begin{array}{c}0.17 \\
\pm 0.03\end{array}$ & $\begin{array}{c}240.4 \\
\pm 6.8\end{array}$ & $\begin{array}{c}0.021 \\
\pm 0.001\end{array}$ & $\begin{array}{c}0.014 \\
\pm 0.001\end{array}$ & $\begin{array}{c}1.54 \\
\pm 0.01\end{array}$ & $\begin{array}{c}6.832 \\
\pm 0.208\end{array}$ & $\begin{array}{c}1.5 \\
\pm 0.28\end{array}$ \\
\hline $04 / 10 / 12$ & 4 & $\begin{array}{c}0.15 \\
\pm 0.02\end{array}$ & $\begin{array}{l}250.6 \\
\pm 12.8\end{array}$ & $\begin{array}{c}0.021 \\
\pm 0.001\end{array}$ & $\begin{array}{c}0.013 \\
\pm 0.001\end{array}$ & $\begin{array}{c}1.62 \\
\pm 0.01\end{array}$ & $\begin{array}{c}6.584 \\
\pm 0.177\end{array}$ & $\begin{array}{c}1.33 \\
\pm 0.20\end{array}$ \\
\hline $04 / 10 / 12$ & 5 & $\begin{array}{c}0.14 \\
\pm 0.02\end{array}$ & $\begin{array}{l}261.1 \\
\pm 33.3\end{array}$ & $\begin{array}{c}0.020 \\
\pm 0.001\end{array}$ & $\begin{array}{c}0.012 \\
\pm 0.001\end{array}$ & $\begin{array}{c}1.73 \\
\pm 0.01\end{array}$ & $\begin{array}{c}6.009 \\
\pm 0.385\end{array}$ & $\begin{array}{c}1.19 \\
\pm 0.21\end{array}$ \\
\hline $13 / 02 / 13$ & 1 & $\begin{array}{c}0.11 \\
\pm 0.01\end{array}$ & $\begin{array}{c}637 \\
\pm 158.5\end{array}$ & $\begin{array}{c}0.010 \\
\pm 0.002\end{array}$ & $\begin{array}{c}0.005 \\
\pm 0.003\end{array}$ & $\begin{array}{c}1.91 \\
\pm 0.01\end{array}$ & $\begin{array}{c}2.459 \\
\pm 0.561\end{array}$ & $\begin{array}{c}0.89 \\
\pm 0.12\end{array}$ \\
\hline $13 / 02 / 13$ & 2 & $\begin{array}{c}0.08 \\
\pm 0.01\end{array}$ & $\begin{array}{l}339.1 \\
\pm 12.8\end{array}$ & $\begin{array}{c}0.014 \\
\pm 0.001\end{array}$ & $\begin{array}{c}0.008 \\
\pm 0.001\end{array}$ & $\begin{array}{c}1.74 \\
\pm 0.01\end{array}$ & $\begin{array}{c}3.847 \\
\pm 0.062\end{array}$ & $\begin{array}{c}0.75 \\
\pm 0.17\end{array}$ \\
\hline $13 / 02 / 13$ & 3 & $\begin{array}{c}0.13 \\
\pm 0.03\end{array}$ & $\begin{array}{l}452.1 \\
\pm 12.2\end{array}$ & $\begin{array}{c}0.013 \\
\pm 0.001\end{array}$ & $\begin{array}{c}0.009 \\
\pm 0.001\end{array}$ & $\begin{array}{c}1.51 \\
\pm 0.01\end{array}$ & $\begin{array}{c}3.556 \\
\pm 0.132\end{array}$ & $\begin{array}{c}1.28 \\
\pm 0.34\end{array}$ \\
\hline $13 / 02 / 13$ & 4 & $\begin{array}{c}0.10 \\
\pm 0.01\end{array}$ & $\begin{array}{l}326.0 \\
\pm 23.8\end{array}$ & $\begin{array}{c}0.016 \\
\pm 0.001\end{array}$ & $\begin{array}{c}0.009 \\
\pm 0.001\end{array}$ & $\begin{array}{c}1.71 \\
\pm 0.01\end{array}$ & $\begin{array}{c}4.179 \\
\pm 0.205\end{array}$ & $\begin{array}{c}1.11 \\
\pm 0.22\end{array}$ \\
\hline $13 / 02 / 13$ & 5 & $\begin{array}{c}0.11 \\
\pm 0.03\end{array}$ & $\begin{array}{l}322.6 \\
\pm 14.3\end{array}$ & $\begin{array}{c}0.016 \\
\pm 0.001\end{array}$ & $\begin{array}{c}0.011 \\
\pm 0.001\end{array}$ & $\begin{array}{c}1.47 \\
\pm 0.01\end{array}$ & $\begin{array}{c}4.236 \\
\pm 0.109\end{array}$ & $\begin{array}{c}1.04 \\
\pm 0.36\end{array}$ \\
\hline $10 / 06 / 13$ & 1 & $\begin{array}{c}0.30 \\
\pm 0.03\end{array}$ & $\begin{array}{l}379.0 \\
\pm 10.8\end{array}$ & $\begin{array}{c}0.020 \\
\pm 0.001\end{array}$ & $\begin{array}{c}0.011 \\
\pm 0.001\end{array}$ & $\begin{array}{c}1.85 \\
\pm 0.01\end{array}$ & $\begin{array}{c}5.849 \\
\pm 0.124\end{array}$ & $\begin{array}{c}1.08 \\
\pm 0.13\end{array}$ \\
\hline $10 / 06 / 13$ & 2 & $\begin{array}{c}0.29 \\
\pm 0.01\end{array}$ & $\begin{array}{l}409.8 \\
\pm 41.9\end{array}$ & $\begin{array}{c}0.019 \\
\pm 0.001\end{array}$ & $\begin{array}{c}0.011 \\
\pm 0.001\end{array}$ & $\begin{array}{c}1.74 \\
\pm 0.01\end{array}$ & $\begin{array}{c}5.640 \\
\pm 0.206\end{array}$ & $\begin{array}{c}1.07 \\
\pm 0.04\end{array}$ \\
\hline $10 / 06 / 13$ & 3 & $\begin{array}{c}0.30 \\
\pm 0.01\end{array}$ & $\begin{array}{l}328.5 \\
\pm 11.7\end{array}$ & $\begin{array}{c}0.020 \\
\pm 0.001\end{array}$ & $\begin{array}{c}0.012 \\
\pm 0.001\end{array}$ & $\begin{array}{c}1.71 \\
\pm 0.01\end{array}$ & $\begin{array}{c}6.188 \\
\pm 0.086\end{array}$ & $\begin{array}{c}1.08 \\
\pm 0.07\end{array}$ \\
\hline $10 / 06 / 13$ & 4 & $\begin{array}{c}0.28 \\
\pm 0.01\end{array}$ & $\begin{array}{l}400.5 \\
\pm 10.5\end{array}$ & $\begin{array}{c}0.016 \\
\pm 0.001\end{array}$ & $\begin{array}{c}0.015 \\
\pm 0.001\end{array}$ & $\begin{array}{c}1.11 \\
\pm 0.01\end{array}$ & $\begin{array}{c}5.162 \\
\pm 0.055\end{array}$ & $\begin{array}{c}1.09 \\
\pm 0.05\end{array}$ \\
\hline $10 / 06 / 13$ & 5 & $\begin{array}{c}0.25 \\
\pm 0.01\end{array}$ & $\begin{array}{l}326.9 \\
\pm 13.2\end{array}$ & $\begin{array}{c}0.018 \\
\pm 0.001\end{array}$ & $\begin{array}{c}0.014 \\
\pm 0.001\end{array}$ & $\begin{array}{c}1.29 \\
\pm 0.01\end{array}$ & $\begin{array}{c}5.832 \\
\pm 0.067\end{array}$ & $\begin{array}{c}1.05 \\
\pm 0.02\end{array}$ \\
\hline $17 / 07 / 13$ & 1 & $\begin{array}{c}0.38 \\
\pm 0.04\end{array}$ & & & & & & $\begin{array}{c}1.51 \\
\pm 0.18\end{array}$ \\
\hline $17 / 07 / 13$ & 2 & $\begin{array}{c}0.25 \\
\pm 0.05\end{array}$ & & & & & & $\begin{array}{c}1.00 \\
\pm 0.20\end{array}$ \\
\hline $17 / 07 / 13$ & 3 & $\begin{array}{c}0.28 \\
\pm 0.05\end{array}$ & & & & & & $\begin{array}{c}1.09 \\
\pm 0.18\end{array}$ \\
\hline $17 / 07 / 13$ & 4 & & & & & & & \\
\hline $17 / 07 / 13$ & 5 & & & & & & & \\
\hline
\end{tabular}


Table S2: Surfactant activity (normalized to Triton T-X-100), total CDOM absorbance (250-450 nm), $\mathrm{S}_{275-295}, \mathrm{~S}_{350-400}, S_{R}, E_{2}$ : $E_{3}$ ratio, salinity and Chl-a of sub-surface water (SSW) from a North Sea transect during 2012-2013.

\begin{tabular}{|c|c|c|c|c|c|c|c|c|c|}
\hline \multirow[b]{2}{*}{ Date } & \multirow{2}{*}{$\begin{array}{c}\text { Dove } \\
\text { Station }\end{array}$} & SA & $\begin{array}{c}\mathrm{CDOM}_{250-} \\
450\end{array}$ & $S_{275-295}$ & $S_{350-400}$ & $S_{R}$ & $E_{2}: E_{3}$ & Chl-a & Salinity \\
\hline & & $\begin{array}{c}\mathrm{mg} \mathrm{l}^{-1} \\
\mathrm{~T}-\mathrm{X}- \\
100\end{array}$ & Total $a$ & $\mathrm{~nm}^{-1}$ & $\mathrm{~nm}^{-1}$ & & \multicolumn{3}{|c|}{$\mathrm{mg} \mathrm{L}^{-1}$} \\
\hline $\begin{array}{c}04 / 10 / 1 \\
2\end{array}$ & 1 & $\begin{array}{c}0.19 \\
\pm 0.05\end{array}$ & $\begin{array}{c}530.5 \\
\pm 0.7\end{array}$ & $\begin{array}{c}0.015 \\
\pm 0.001\end{array}$ & $\begin{array}{c}0.011 \\
\pm 0.001\end{array}$ & $\begin{array}{c}1.37 \\
\pm 0.01\end{array}$ & $\begin{array}{c}4.21 \\
\pm 0.04\end{array}$ & $\begin{array}{c}0.55 \\
\pm 0.03\end{array}$ & 33.1 \\
\hline $\begin{array}{c}04 / 10 / 1 \\
2\end{array}$ & 2 & $\begin{array}{c}0.12 \\
\pm 0.01\end{array}$ & $\begin{array}{c}241.5 \\
\pm 5.5\end{array}$ & $\begin{array}{c}0.020 \\
\pm 0.001\end{array}$ & $\begin{array}{c}0.013 \\
\pm 0.001\end{array}$ & $\begin{array}{c}1.59 \\
\pm 0.01\end{array}$ & $\begin{array}{c}6.44 \\
\pm 0.19\end{array}$ & $\begin{array}{c}0.53 \\
\pm 0.01\end{array}$ & 34.0 \\
\hline $\begin{array}{c}04 / 10 / 1 \\
2\end{array}$ & 3 & $\begin{array}{c}0.12 \\
\pm 0.01\end{array}$ & $\begin{array}{c}240.0 \\
\pm 3.5\end{array}$ & $\begin{array}{c}0.021 \\
\pm 0.001\end{array}$ & $\begin{array}{c}0.013 \\
\pm 0.001\end{array}$ & $\begin{array}{c}1.60 \\
\pm 0.01\end{array}$ & $\begin{array}{c}6.66 \\
\pm 0.08\end{array}$ & $\begin{array}{c}1.05 \\
\pm 0.02\end{array}$ & 34.0 \\
\hline $\begin{array}{c}04 / 10 / 1 \\
2\end{array}$ & 4 & $\begin{array}{c}0.11 \\
\pm 0.01\end{array}$ & $\begin{array}{c}227.2 \\
\pm 3.8\end{array}$ & $\begin{array}{c}0.021 \\
\pm 0.001\end{array}$ & $\begin{array}{c}0.013 \\
\pm 0.001\end{array}$ & $\begin{array}{c}1.63 \\
\pm 0.01\end{array}$ & $\begin{array}{c}6.60 \\
\pm 0.11\end{array}$ & $\begin{array}{c}1.54 \\
\pm 0.04\end{array}$ & 34.2 \\
\hline $\begin{array}{c}04 / 10 / 1 \\
2\end{array}$ & 5 & $\begin{array}{c}0.12 \\
\pm 0.01\end{array}$ & $\begin{array}{c}220.3 \\
\pm 0.8\end{array}$ & $\begin{array}{c}0.021 \\
\pm 0.001\end{array}$ & $\begin{array}{c}0.013 \\
\pm 0.001\end{array}$ & $\begin{array}{c}1.65 \\
\pm 0.01\end{array}$ & $\begin{array}{c}6.74 \\
\pm 0.08\end{array}$ & $\begin{array}{c}1.00 \\
\pm 0.02\end{array}$ & 34.1 \\
\hline $\begin{array}{c}13 / 02 / 1 \\
3\end{array}$ & 1 & $\begin{array}{c}0.12 \\
\pm 0.01\end{array}$ & $\begin{array}{l}792.2 \\
\pm 10.7\end{array}$ & $\begin{array}{c}0.009 \\
\pm 0.001\end{array}$ & $\begin{array}{c}0.005 \\
\pm 0.001\end{array}$ & $\begin{array}{c}1.80 \\
\pm 0.01\end{array}$ & $\begin{array}{c}2.25 \\
\pm 0.10\end{array}$ & $\begin{array}{c}0.35 \\
\pm 0.02\end{array}$ & 33.8 \\
\hline $\begin{array}{c}13 / 02 / 1 \\
3\end{array}$ & 2 & $\begin{array}{c}0.10 \\
\pm 0.02\end{array}$ & $\begin{array}{l}376.0 \\
\pm 34.3\end{array}$ & $\begin{array}{c}0.014 \\
\pm 0.001\end{array}$ & $\begin{array}{c}0.007 \\
\pm 0.001\end{array}$ & $\begin{array}{c}1.93 \\
\pm 0.01\end{array}$ & $\begin{array}{c}3.42 \\
\pm 0.25\end{array}$ & $\begin{array}{c}0.15 \\
\pm 0.02\end{array}$ & 33.9 \\
\hline $\begin{array}{c}13 / 02 / 1 \\
3\end{array}$ & 3 & $\begin{array}{c}0.10 \\
\pm 0.01\end{array}$ & $\begin{array}{c}478.3 \\
\pm 9.9\end{array}$ & $\begin{array}{c}0.013 \\
\pm 0.001\end{array}$ & $\begin{array}{c}0.008 \\
\pm 0.001\end{array}$ & $\begin{array}{c}1.65 \\
\pm 0.01\end{array}$ & $\begin{array}{c}3.30 \\
\pm 0.01\end{array}$ & $\begin{array}{c}0.14 \\
\pm 0.01\end{array}$ & 33.5 \\
\hline $\begin{array}{c}13 / 02 / 1 \\
3\end{array}$ & 4 & $\begin{array}{c}0.09 \\
\pm 0.02\end{array}$ & $\begin{array}{l}377.1 \\
\pm 24.4\end{array}$ & $\begin{array}{c}0.017 \\
\pm 0.001\end{array}$ & $\begin{array}{c}0.008 \\
\pm 0.001\end{array}$ & $\begin{array}{c}2.14 \\
\pm 0.01\end{array}$ & $\begin{array}{c}3.95 \\
\pm 0.03\end{array}$ & $\begin{array}{c}0.09 \\
\pm 0.03\end{array}$ & 33.9 \\
\hline $\begin{array}{c}13 / 02 / 1 \\
3\end{array}$ & 5 & $\begin{array}{c}0.11 \\
\pm 0.03\end{array}$ & $\begin{array}{c}292.4 \\
\pm 2.3\end{array}$ & $\begin{array}{c}0.016 \\
\pm 0.001\end{array}$ & $\begin{array}{c}0.009 \\
\pm 0.001\end{array}$ & $\begin{array}{c}1.80 \\
\pm 0.01\end{array}$ & $\begin{array}{c}4.32 \\
\pm 0.10\end{array}$ & $\begin{array}{c}0.11 \\
\pm 0.01\end{array}$ & 33.7 \\
\hline $\begin{array}{c}10 / 06 / 1 \\
3\end{array}$ & 1 & $\begin{array}{c}0.27 \\
\pm 0.01\end{array}$ & $\begin{array}{c}322.8 \\
\pm 2.1\end{array}$ & $\begin{array}{c}0.020 \\
\pm 0.001\end{array}$ & $\begin{array}{c}0.009 \\
\pm 0.001\end{array}$ & $\begin{array}{c}2.25 \\
\pm 0.01\end{array}$ & $\begin{array}{c}5.93 \\
\pm 0.04\end{array}$ & $\begin{array}{c}0.65 \\
\pm 0.04\end{array}$ & 33.6 \\
\hline $\begin{array}{c}10 / 06 / 1 \\
3\end{array}$ & 2 & $\begin{array}{c}0.28 \\
\pm 0.01\end{array}$ & $\begin{array}{c}284.1 \\
\pm 3.2\end{array}$ & $\begin{array}{c}0.021 \\
\pm 0.001\end{array}$ & $\begin{array}{c}0.010 \\
\pm 0.001\end{array}$ & $\begin{array}{c}2.20 \\
\pm 0.01\end{array}$ & $\begin{array}{c}6.55 \\
\pm 0.08\end{array}$ & $\begin{array}{c}0.61 \\
\pm 0.09\end{array}$ & 34.1 \\
\hline $\begin{array}{c}10 / 06 / 1 \\
3\end{array}$ & 3 & $\begin{array}{c}0.28 \\
\pm 0.01\end{array}$ & $\begin{array}{c}261.9 \\
\pm 0.8\end{array}$ & $\begin{array}{c}0.022 \\
\pm 0.001\end{array}$ & $\begin{array}{c}0.011 \\
\pm 0.001\end{array}$ & $\begin{array}{c}2.02 \\
\pm 0.01\end{array}$ & $\begin{array}{c}7.05 \\
\pm 0.06\end{array}$ & $\begin{array}{c}0.36 \\
\pm 0.02\end{array}$ & 34.0 \\
\hline $\begin{array}{c}10 / 06 / 1 \\
3\end{array}$ & 4 & $\begin{array}{c}0.26 \\
\pm 0.01\end{array}$ & $\begin{array}{c}253.8 \\
\pm 1.0\end{array}$ & $\begin{array}{c}0.021 \\
\pm 0.001\end{array}$ & $\begin{array}{c}0.011 \\
\pm 0.001\end{array}$ & $\begin{array}{c}1.95 \\
\pm 0.01\end{array}$ & $\begin{array}{c}7.26 \\
\pm 0.11\end{array}$ & $\begin{array}{c}0.33 \\
\pm 0.01\end{array}$ & 34.2 \\
\hline $\begin{array}{c}10 / 06 / 1 \\
3\end{array}$ & 5 & $\begin{array}{c}0.24 \\
\pm 0.01\end{array}$ & $\begin{array}{c}217.2 \\
\pm 1.2\end{array}$ & $\begin{array}{c}0.023 \\
\pm 0.001\end{array}$ & $\begin{array}{c}0.013 \\
\pm 0.001\end{array}$ & $\begin{array}{c}1.76 \\
\pm 0.01\end{array}$ & $\begin{array}{c}8.43 \\
\pm 0.14\end{array}$ & $\begin{array}{c}0.22 \\
\pm 0.07\end{array}$ & 34.2 \\
\hline $\begin{array}{c}17 / 07 / 1 \\
3\end{array}$ & 1 & $\begin{array}{c}0.25 \\
\pm 0.01\end{array}$ & & & & & & $\begin{array}{c}0.12 \\
\pm 0.10\end{array}$ & 34.6 \\
\hline $\begin{array}{c}17 / 07 / 1 \\
3\end{array}$ & 2 & $\begin{array}{c}0.25 \\
\pm 0.01\end{array}$ & & & & & & $\begin{array}{c}0.35 \\
\pm 0.06\end{array}$ & 34.4 \\
\hline $\begin{array}{c}17 / 07 / 1 \\
3\end{array}$ & 3 & $\begin{array}{c}0.25 \\
\pm 0.01\end{array}$ & & & & & & $\begin{array}{c}0.38 \\
\pm 0.08\end{array}$ & 34.3 \\
\hline $\begin{array}{c}17 / 07 / 1 \\
3\end{array}$ & 4 & & & & & & & $\begin{array}{c}0.12 \\
\pm 0.01\end{array}$ & 34.5 \\
\hline $\begin{array}{c}17 / 07 / 1 \\
3\end{array}$ & 5 & & & & & & & $\begin{array}{c}0.14 \\
\pm 0.01\end{array}$ & 34.6 \\
\hline
\end{tabular}


Table S3: $k_{660}$ and $\mathrm{R}_{660}$ estimates for three water-side turbulence settings of $0.6,0.7$ and $0.75 \mathrm{~Hz}$ from North East coast transect 2012-2013

\begin{tabular}{|c|c|c|c|c|c|c|c|}
\hline \multirow{2}{*}{ Date } & \multirow{2}{*}{$\begin{array}{c}\text { Dove } \\
\text { Station }\end{array}$} & \multicolumn{3}{|c|}{$\mathrm{CH}_{4} k_{660}$} & \multicolumn{3}{|c|}{$\mathrm{CH}_{4} R_{660}$} \\
\hline & & $0.6 \mathrm{~Hz}$ & $0.7 \mathrm{~Hz}$ & $0.75 \mathrm{~Hz}$ & $0.6 \mathrm{~Hz}$ & $0.7 \mathrm{~Hz}$ & $0.75 \mathrm{~Hz}$ \\
\hline $04 / 10 / 12$ & 1 & $9.58 \pm 0.52$ & $11.21 \pm 0.45$ & $11.40 \pm 0.28$ & $0.60 \pm 0.03$ & $0.70 \pm 0.03$ & $0.72 \pm 0.02$ \\
\hline $04 / 10 / 12$ & 2 & $9.39 \pm 0.48$ & $11.51 \pm 0.46$ & $11.02 \pm 0.26$ & $0.59 \pm 0.03$ & $0.72 \pm 0.03$ & $0.69 \pm 0.02$ \\
\hline $04 / 10 / 12$ & 3 & $9.58 \pm 0.52$ & $12.62 \pm 0.52$ & $11.25 \pm 0.29$ & $0.60 \pm 0.03$ & $0.79 \pm 0.03$ & $0.71 \pm 0.02$ \\
\hline $04 / 10 / 12$ & 4 & $10.50 \pm 0.40$ & $12.32 \pm 0.49$ & $10.66 \pm 0.31$ & $0.66 \pm 0.02$ & $0.77 \pm 0.03$ & $0.67 \pm 0.02$ \\
\hline $04 / 10 / 12$ & 5 & $12.25 \pm 0.55$ & $13.59 \pm 0.57$ & $12.77 \pm 0.34$ & $0.77 \pm 0.03$ & $0.85 \pm 0.04$ & $0.80 \pm 0.02$ \\
\hline $13 / 02 / 13$ & 1 & $12.17 \pm 0.56$ & $12.79 \pm 0.55$ & $12.72 \pm 0.35$ & $0.76 \pm 0.04$ & $0.80 \pm 0.03$ & $0.80 \pm 0.02$ \\
\hline $13 / 02 / 13$ & 2 & & & & & & \\
\hline $13 / 02 / 13$ & 3 & $12.68 \pm 0.66$ & $13.67 \pm 0.56$ & $11.16 \pm 0.42$ & $0.80 \pm 0.04$ & $0.86 \pm 0.04$ & $0.70 \pm 0.03$ \\
\hline $13 / 02 / 13$ & 4 & $10.97 \pm 0.46$ & $13.21 \pm 0.53$ & $12.55 \pm 0.34$ & $0.69 \pm 0.03$ & $0.83 \pm 0.03$ & $0.79 \pm 0.02$ \\
\hline $13 / 02 / 13$ & 5 & $13.04 \pm 0.51$ & $13.6 \pm 0.54$ & $13.11 \pm 0.37$ & $0.82 \pm 0.03$ & $0.85 \pm 0.03$ & $0.82 \pm 0.02$ \\
\hline $10 / 06 / 13$ & 1 & $8.17 \pm 0.37$ & $9.05 \pm 0.40$ & $8.70 \pm 0.44$ & $0.51 \pm 0.02$ & $0.57 \pm 0.02$ & $0.55 \pm 0.03$ \\
\hline $10 / 06 / 13$ & 2 & $7.77 \pm 0.33$ & $8.93 \pm 0.37$ & $8.65 \pm 0.25$ & $0.49 \pm 0.02$ & $0.56 \pm 0.02$ & $0.54 \pm 0.02$ \\
\hline $10 / 06 / 13$ & 3 & $8.02 \pm 0.33$ & $10.46 \pm 0.43$ & $11.54 \pm 0.42$ & $0.50 \pm 0.02$ & $0.66 \pm 0.03$ & $0.72 \pm 0.03$ \\
\hline $10 / 06 / 13$ & 4 & & & & & & \\
\hline $10 / 06 / 13$ & 5 & $9.51 \pm 0.38$ & $10.52 \pm 0.44$ & $9.83 \pm 0.26$ & $0.60 \pm 0.02$ & $0.66 \pm 0.03$ & $0.62 \pm 0.02$ \\
\hline $17 / 07 / 13$ & 1 & $9.71 \pm 0.41$ & $10.92 \pm 0.47$ & $10.13 \pm 0.36$ & $0.61 \pm 0.03$ & $0.69 \pm 0.03$ & $0.64 \pm 0.02$ \\
\hline $17 / 07 / 13$ & 2 & $10.29 \pm 0.54$ & $9.94 \pm 0.47$ & $9.50 \pm 0.24$ & $0.65 \pm 0.03$ & $0.62 \pm 0.03$ & $0.60 \pm 0.02$ \\
\hline $17 / 07 / 13$ & 3 & $10.02 \pm 0.62$ & $11.15 \pm 0.48$ & $7.91 \pm 3.90$ & $0.63 \pm 0.04$ & $0.70 \pm 0.03$ & $0.50 \pm 0.02$ \\
\hline $17 / 07 / 13$ & 4 & & & & & & \\
\hline $17 / 07 / 13$ & 5 & & & & & & \\
\hline
\end{tabular}

